\title{
IL-7 receptor deletion ameliorates diet-induced obesity and insulin resistance in mice
}

\author{
Miyoung Lee ${ }^{1} \cdot$ Su Jin Song ${ }^{1} \cdot$ Myung-Sook Choi $^{2} \cdot$ Rina Yu $^{3} \cdot$ Taesun Park $^{1}$
}

Received: 3 December 2014 / Accepted: 17 June 2015 /Published online: 8 July 2015

(C) Springer-Verlag Berlin Heidelberg 2015

\begin{abstract}
Aim/hypothesis Obesity-induced inflammation plays an important role in the development of insulin resistance and type 2 diabetes. Recent studies have demonstrated that adiposity can be improved by ablating certain inflammatory signalling pathways. Although the IL-7 receptor (IL-7R) is mostly known as a key regulator of $\mathrm{T}$ lymphocyte development and homeostasis, its role in obesity and metabolic diseases is unknown. Because IL-7 is markedly increased in the serum of obese individuals and $I L-7 R$ (also known as $I L 7 R$ ) is overexpressed in white adipose tissue (WAT) in obesity, we studied the metabolic consequences of genetic $1 l-7 r$ ablation in mice.

Methods Age-matched Il-7r-deficient (Il-7r KO) and wildtype (WT) littermates were fed a standard chow or high-fat diet (HFD) for 14 weeks. Their serum metabolic variables were measured. The expression of genes and proteins related to insulin resistance and inflammation was evaluated in WAT. Results We demonstrated that $I l-7 r \mathrm{KO}$ mice exhibited significantly reduced body weight gain and visceral adiposity
\end{abstract}

Electronic supplementary material The online version of this article (doi:10.1007/s00125-015-3684-7) contains peer-reviewed but unedited supplementary material, which is available to authorised users.

Taesun Park

tspark@yonsei.ac.kr

1 Department of Food and Nutrition, Brain Korea 21 Plus Project, Yonsei University, 50 Yonsei-ro, Seodaemun-gu, Seoul 120-749, Republic of Korea

2 Department of Food Science and Nutrition, Kyungpook National University, Daegu, Republic of Korea

3 Department of Food Science and Nutrition, University of Ulsan, Mugeo-dong, Nam-ku, Ulsan, Republic of Korea compared with WT controls on both chow and HFD. The expression of signalling molecules involved in adipogenesis was reduced in the WAT of $I l-7 r \mathrm{KO}$ mice. We also found that Il-7r KO mice had significantly enhanced glucose homeostasis and insulin sensitivity. Consistent with an improved metabolic phenotype, proinflammatory cytokine production and macrophage infiltration was attenuated in the WAT of $I l-7 r$ $\mathrm{KO}$ mice.

Conclusions/interpretation The IL-7R plays an important role in the induction of HFD-induced adipogenesis and insulin resistance in mice.

Keywords Adipogenesis · Inflammation $\cdot$ Insulin resistance · Interleukin-7 receptor · Obesity
Abbreviations
DAB Diaminobenzidine
epiWAT Epididymal WAT
ERK Extracellular signal-regulated kinase
HFD High-fat diet
IKK $\beta \quad$ Inhibitor of $\kappa B$ kinase $\beta$
IL-7R IL-7 receptor
JAK Janus kinase
JNK c-Jun amino-terminal kinase
MAPK Mitogen-activated protein kinase
MCP-1 Monocyte chemoattractant protein-1
PI3K Phosphatidylinositol 3-kinase
SOCS Suppressor of cytokine signalling
STAT5 Signal transducer and activator of transcription 5
subWAT Subcutaneous WAT
WAT White adipose tissue
WT Wild-type 


\section{Introduction}

The IL-7 ligand receptor system plays an important role in the normal development and homeostatic maintenance of the immune system [1]. Ligand-receptor interaction leads to a cascade of phosphorylation events mediated by signalling molecules, including Janus kinase (JAK)-1 and -3, signal transducer and activator of transcription 5 (STAT5), and phosphatidylinositol 3-kinase (PI3K) [2, 3]. The physiological result of IL-7 receptor (IL-7R) activation is survival, proliferation and maturation in early B lymphocytes and induction of the activation cascade in mature $\mathrm{T}$ lymphocytes [4]. IL-7R is a dimeric complex composed of a high affinity $\alpha$ chain and the common $\gamma$ chain, which is shared with several other cytokine receptors, namely IL-2, IL-4, IL-9, IL-15 and IL-21 [5]. Mutations of the gene encoding IL-7R $\alpha$ are associated with a milder form of severe combined immunodeficiency disease, which is characterised by a specific lack of $\mathrm{T}$ cells $[6,7]$.

Obesity is now recognised as a state of chronic low-grade systemic inflammation [8]. Adipose tissue, particularly the visceral fat of obese humans and rodents, is affected by this inflammatory state, which is characterised by increased production and secretion of proinflammatory molecules $[9,10]$. Many lines of evidence have shown that the activation of proinflammatory pathways within insulin target tissues can impair insulin signalling $[11,12]$. TNF- $\alpha$ was among the first mediators defined as a key factor linking inflammation and insulin resistance [13]. Engagement of the TNF receptor with TNF- $\alpha$ induces inhibitory phosphorylation of serine residues of IRS 1 and activates the inhibitor of $\kappa B$ kinase $\beta(\operatorname{IKK} \beta)$ and c-Jun amino-terminal kinase (JNK) pathways, two major intracellular regulators of insulin resistance $[14,15]$. Several studies have reported that IL-6 mediates insulin resistance in the obese state by inducing suppressor of cytokine signalling (SOCS)-1 and -3, which link IRS1 to ubiquitin-mediated degradation $[16,17]$. Accumulating evidence reveals that IL-7 induces the production of proinflammatory cytokines, such as TNF- $\alpha$ and IL- 6 , in monocytes and macrophages [18, 19]. Chronic activation of the IL-7/IL-7R signalling pathway contributes to persistent inflammation associated with inflammatory pathologies, including atherosclerosis, arthritis, gastritis and colitis in rodent models [19-23]. Recently, IL-7 expression was reported to be upregulated in the adipose tissue of obese individuals [24], supporting the notion that IL-7 could be a potential mediator of obesity-associated inflammation. The aim of this study was to investigate whether IL-7R plays a role in the induction of both adiposity and insulin resistance. Therefore, we assessed the effects of whole-body deletion of $I l-7 r$ (also known as $I l 7 r$ ) on visceral adiposity and glucose homeostasis in mice.

\section{Methods}

Animal and diets C57BL/6J wild-type (WT; stock 000664)

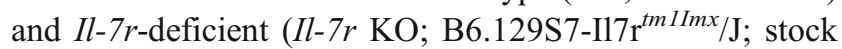
002295) mice were obtained from the Jackson Laboratory (Bar Harbor, ME, USA). $I l-7 r$ KO mice were backcrossed to a C57BL/6J background for six generations. At 5 weeks of age, WT and $I l-7 r$ KO mice were separated by sex and placed on either a chow diet (Purina Rodent Chow 5001, Orient Bio, Seongnam, Gyeonggi-do, South Korea) or high-fat diet (HFD) for 14 weeks. The HFD was composed of $200 \mathrm{~g}$ of fat $/ \mathrm{kg}$ (170 $\mathrm{g}$ of lard plus $30 \mathrm{~g}$ of corn oil) and $1 \%$ cholesterol. All mice were housed in standard cages in a temperature controlled $\left(21 \pm 2.0^{\circ} \mathrm{C}\right)$ room with a $12 \mathrm{~h}$ light-dark cycle and were given free access to purified water.

Food intake of the mice was recorded daily and their body weights were measured weekly. At the end of the experimental period, after being fasted for $16 \mathrm{~h}$, the mice were anaesthetised with diethyl ether and their plasma was obtained and stored at $-80^{\circ} \mathrm{C}$. The visceral and subcutaneous fat pads were dissected out, weighed, snap frozen in liquid nitrogen and then stored at $-80^{\circ} \mathrm{C}$. All animal experiments were performed in accordance with the Korean Food and Drug Administration guidelines. Protocols were reviewed and approved by the Institutional Animal Care and Use Committee of the Yonsei Laboratory Animal Research Center (YLARC) (Permit no. 2011-0061). All mice were maintained in the YLARC's pathogen-free facility.

Biochemical analysis Plasma concentrations of triacylglycerol, total cholesterol, NEFA, HDL-cholesterol and glucose were measured using commercial kits (Bio-Clinical System, Gyeonggi-do, Republic of Korea). Plasma insulin levels were analysed using a mouse insulin ELISA kit (Alpco Diagnostics, Windham, NH, USA). The HOMA-IR index was calculated as fasting plasma glucose concentration ( $\mathrm{mmol} / \mathrm{l})$ multiplied by fasting insulin level (pmol/l) divided by 22.5. Plasma levels of TNF- $\alpha$, IL- 6 and monocyte chemoattractant protein1 (MCP-1) were measured using an ELISA kit (Millipore, Billerica, MA, USA).

Histological analysis The epididymal fat pads were fixed in neutral buffered formalin, embedded in paraffin and sectioned at $5 \mu \mathrm{m}$. The tissue sections were stained with hematoxylin and eosin (H\&E).

Oral glucose tolerance test Two weeks before the end of the experimental period, mice were fasted for $16 \mathrm{~h}$ and administered orally with glucose (gavage with $2 \mathrm{~g}$ glucose/kg body weight). Blood glucose was measured using a Precision Q.I.D glucometer (Abbott Laboratories, North Chicago, IL, USA). 
RNA extraction and semi-quantitative RT-PCR Total RNA was isolated from the epididymal white adipose tissue (epiWAT) and subcutaneous WAT (subWAT) of each mouse using Trizol reagent (Invitrogen, Carlsbad, CA, USA) according to the manufacturer's instructions and was reverse transcribed using the Superscript II kit (Invitrogen) according to the manufacturer's recommendations. PCR was performed as follows: $10 \mathrm{~min}$ at $94^{\circ} \mathrm{C} ; 30-33$ cycles at $94^{\circ} \mathrm{C}$ for $30 \mathrm{~s}$ and $55^{\circ} \mathrm{C}$ for $30 \mathrm{~s} ; 72^{\circ} \mathrm{C}$ for $1 \mathrm{~min}$ and $10 \mathrm{~min}$ incubation at $72^{\circ} \mathrm{C}$. PCR products were size-fractionated on a $2 \%$ agarose gel and stained with ethidium bromide. The band intensities were quantified using Quantity One analysis software 4.6 (BioRad, Hercules, CA, USA). Sequences of all primers are listed in electronic supplementary material (ESM) Table 1.

Western blot analysis To obtain total protein, the epiWAT samples obtained from each mouse were homogenised at $4{ }^{\circ} \mathrm{C}$ in an extraction buffer (composition in mmol/l: 100 Tris- $\mathrm{HCl}, \mathrm{pH} 7.4,5$ EDTA, $50 \mathrm{NaCl}, 50$ sodium pyrophosphate, $50 \mathrm{NaF}$ and 100 orthovanadate plus $1 \%$ Triton X-100, $1 \mathrm{mmol} / 1$ phenylmethanesulfonyl fluoride, $2 \mu \mathrm{g} / \mathrm{ml}$ aprotinin, $1 \mu \mathrm{g} / \mathrm{ml}$ pepstatin A and $1 \mu \mathrm{g} / \mathrm{ml}$ leupeptin) and centrifuged at $13,000 \mathrm{~g}$ for $20 \mathrm{~min}$ at $4^{\circ} \mathrm{C}$. To obtain membrane protein, the epiWAT was homogenised in a buffer containing $20 \mathrm{mmol} / 1$ HEPES, pH 7.4, 4 mmol/l EDTA, $250 \mathrm{mmol} / 1$ sucrose, two tablets of protein inhibitor, $1 \mathrm{mmol} / \mathrm{l}$ sodium orthovanadate and $1 \%$ Triton $\mathrm{X}-100$. The homogenates were centrifuged at $2,000 \mathrm{~g}$ for $1.5 \mathrm{~h}$ at $4^{\circ} \mathrm{C}$ and the supernatant fractions were centrifuged at $150,000 \mathrm{~g}$ for $1.5 \mathrm{~h}$ at $4^{\circ} \mathrm{C}$. Total protein or membrane protein $(40 \mu \mathrm{g})$ was separated on an $8 \%$ SDSPAGE gel and subsequently transferred onto nitrocellulose membranes (Amersham, Little Chalfont, UK). The membranes were blocked with 5\% BSA in Tris-buffered saline/ Tween buffer (10 mmol/1 Tris- $\mathrm{HCl}, \mathrm{pH} 7.5,150 \mathrm{mmol} / \mathrm{l} \mathrm{NaCl}$ and $0.05 \%$ Tween 20 ), incubated with primary antibodies (diluted $1: 1,000)$ overnight at $4^{\circ} \mathrm{C}$ and washed in Tris-buffered saline with $0.05 \%$ Tween 20 . Antibodies to the following proteins were commercially obtained from the indicated sources: $\beta$-actin, STAT5, phospho-STAT5 and SOCS-1 from Santa Cruz Biotechnology (Santa Cruz, CA, USA) and extracellular signal-regulated kinase (ERK), phospho-ERK (Thr202/Tyr204), IRS1, phospho-IRS1 (Tyr20), protein kinase B (Akt), phospho-Akt (Tyr176) and GLUT4 from Cell Signaling Technology (Danvers, MA, USA). After washing, the corresponding secondary antibodies were applied for $1 \mathrm{~h}$ at room temperature in Tris-buffered saline containing $0.05 \%$ Tween-20. Signals were detected using a chemiluminescent detection system (Amersham). The protein bands were then quantified using Quantity One analysis software 4.6 (Bio-Rad).

Immunohistochemistry Paraformaldehyde-fixed, paraffinembedded epiWAT was cut into $4 \mu \mathrm{m}$ sections. For antigen retrieval, the slides were autoclaved in $10 \mathrm{mmol} / \mathrm{l}$ sodium citrate buffer ( $\mathrm{pH}$ 6.0). Endogenous peroxidase was blocked with $0.345 \% \mathrm{H}_{2} \mathrm{O}_{2}$ for $30 \mathrm{~min}$ and sections were further blocked with 5\% BSA in PBS for $30 \mathrm{~min}$. The sections were incubated overnight at $4{ }^{\circ} \mathrm{C}$ with the primary antibody $\mathrm{F} 4 / 80$ (1:100) (Abcam, Cambridge, MA, USA) and then incubated with biotinylated anti-mouse secondary antibody $(1: 200)$ (Dako, Glostrup, Denmark) for $1 \mathrm{~h}$ at room temperature, followed by incubation in avidin-biotin complex solution (Vector, Burlingame, CA, USA). Staining was performed with diaminobenzidine (DAB) (Vector) according to the manufacturer's protocol. The DAB-stained preparations were visualised with a general optical microscope, TE-2000 (Nikon, Tokyo, Japan).

Statistical analysis RT-PCR and Western blotting data are shown as the mean \pm SEM of three independent experiments ( $n=2$ or 3 per experiment) for each group, cumulatively including eight mice. An unpaired Student's $t$ test analysis was used for all data comparisons between the $I l-7 r \mathrm{KO}$ and WT control mice fed either chow or an HFD. All statistical analyses were performed with SPSS 21.0 software (SPSS, Chicago, IL, USA), and significance was set at $* p<0.05, * * p<0.01$ and $* * * p<0.001$.

\section{Results}

HFD increased $I l-7 r$ expression in the WAT of mice $I l-7 r$ mRNA was expressed in the epiWAT of WT mice fed both chow and HFD, with levels being significantly higher in the HFD-fed mice (Fig. 1a). As expected, $\mathrm{Il}-7 r \mathrm{KO}$ mice did not express $I l-7 r$ mRNA (Fig. 1a). Expression of phosphorylated STAT5 (p-STAT5) and SOCS-1 was downregulated in the epiWAT of $I l-7 r \mathrm{KO}$ mice relative to WT controls (Fig. 1b, c).

Il-7r KO mice exhibited decreased body weight and visceral fat pad weight To evaluate the function of IL-7R in energy metabolism, male age-matched $\mathrm{WT}$ and $\mathrm{Il}-7 r \mathrm{KO}$ mice were fed with normal chow or HFD for 14 weeks. On both chow and HFD, food intake did not differ between $I l-7 r \mathrm{KO}$ mice and WT controls (Fig. 2c). Il-7r KO mice showed significantly decreased final body weight and cumulative body weight gain compared with WT controls on HFD (Fig. 2a, b). The food efficiency ratio in $\mathrm{Il}-7 r \mathrm{KO}$ mice was significantly lower than in WT mice under both chow and HFD conditions (Fig. 2d). Il-7r KO mice also had significantly decreased total visceral fat pad weight compared with WT controls fed both chow and HFD (Fig. 2e). H\&E staining showed decreased adipocyte size in the epididymal fat of $I l-7 r \mathrm{KO}$ vs WT mice (Fig. 2f). Female $\mathrm{Il}-7 r \mathrm{KO}$ mice also showed decreased body weight, body weight gain, food efficiency ratio, total visceral fat pad weight and adipocyte size compared with WT controls fed both chow and HFD (ESM Fig. 1). 
$\mathbf{a}$
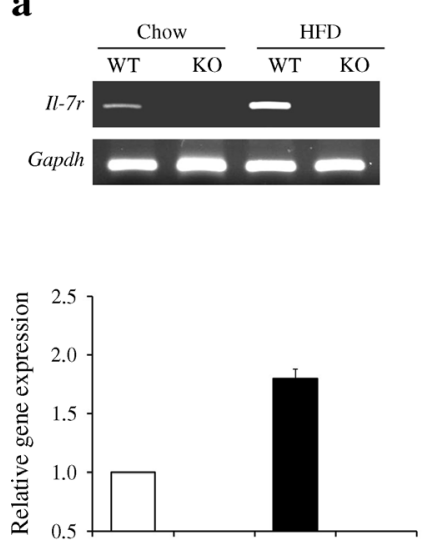

b
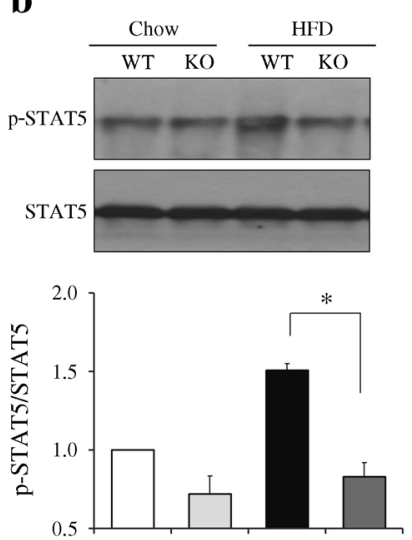

c
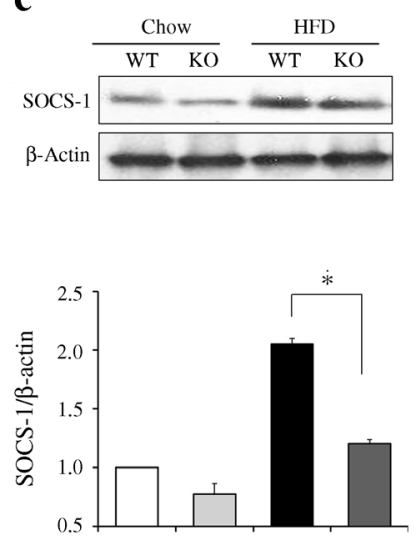

Fig. 1 HFD increases $I l-7 r$ expression in the adipose tissue of mice. (a) Levels of $I l-7 r$ mRNA in the epiWAT from WT and $I l-7 r$ KO mice. (b, c) WT and $l l-7 r \mathrm{KO}$ adipose tissue protein lysates were probed for levels of phosphorylated STAT5, SOCS-1 and $\beta$-actin by immunoblot analysis.

\section{Il-7r KO mice showed decreased plasma lipid levels Plasma} concentrations of triacylglycerol, NEFA, and total cholesterol were significantly lower in male $I l-7 r \mathrm{KO}$ mice than in their
Data are means \pm SEM, $n=8 .{ }^{*} p<0.05, I l-7 r$ KO vs WT. White bars, WT chow; black bars, WT HFD; light grey bars, $I l-7 r$ KO chow; dark grey bars, $I l-7 r$ KO HFD

WT controls on both chow and HFD (Fig. 3a-c). There was no difference in HDL-cholesterol levels between $\mathrm{Il}-7 r \mathrm{KO}$ mice and WT controls (Fig. 3d). Decreased plasma lipid levels were

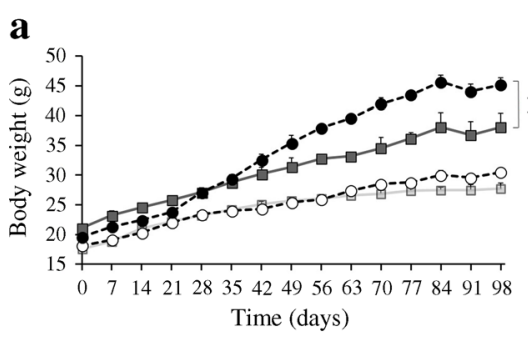

b

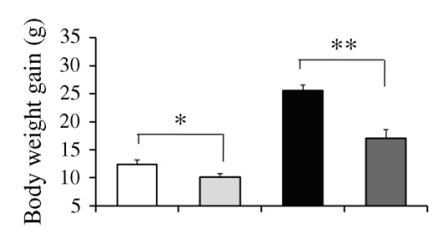

c

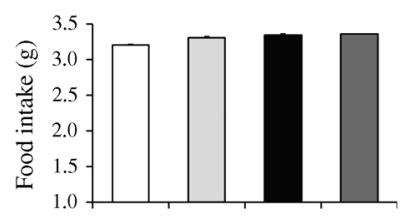

d

$\mathbf{e}$
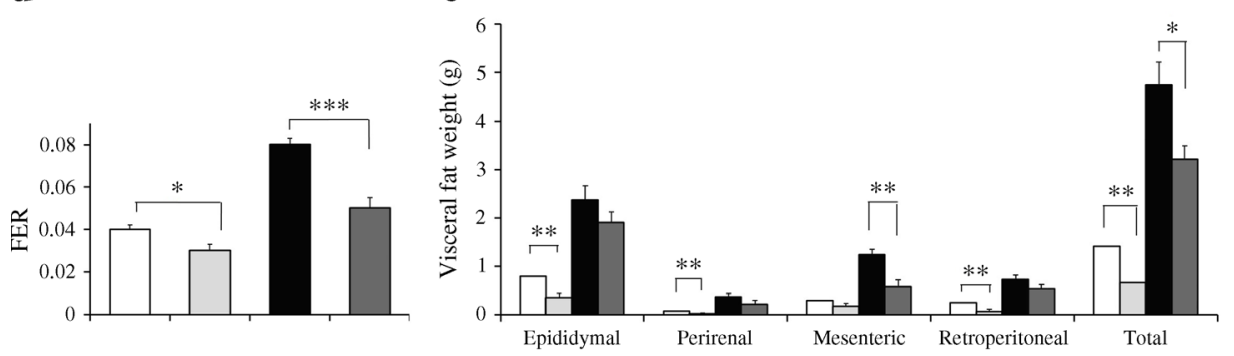

f WT $\mathrm{KO}$

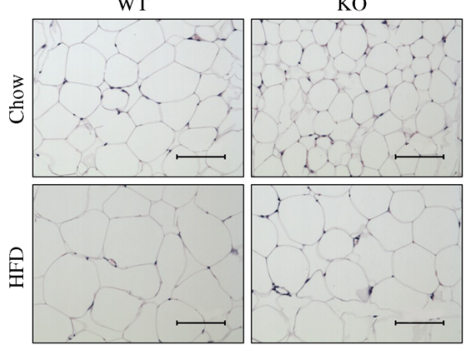

g

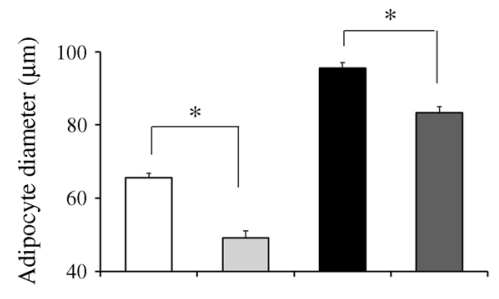

Fig. 2 Deletion of $I l-7 r$ reduces body weight gain and visceral adiposity. (a) Body weight of male WT and $I l-7 r$ KO mice fed chow or HFD for 14 weeks. (b) Body weight gain. (c) Food intake. (d) Food efficiency ratio (FER; body weight gain over the experimental period [g]/food intake over the experimental period $[\mathrm{g}]$ ). (e) Visceral fat pad weights. (f) Representa-

(g) Size of adipocytes within epiWAT. Data are means \pm SEM, $n=8$. ${ }^{*} p<0.05,{ }^{*} p<0.01$ and ${ }^{* * *} p<0.001, I l-7 r \mathrm{KO}$ vs WT. White circles and bars, WT chow; black circles and bars, WT HFD; light grey squares and bars, $I l-7 r$ KO chow; dark grey squares and bars, $I l-7 r$ KO HFD 


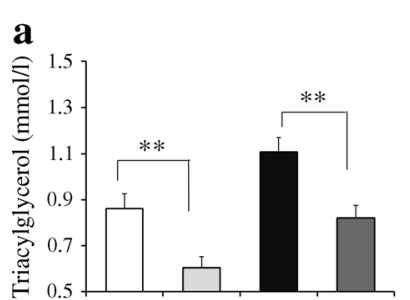

b
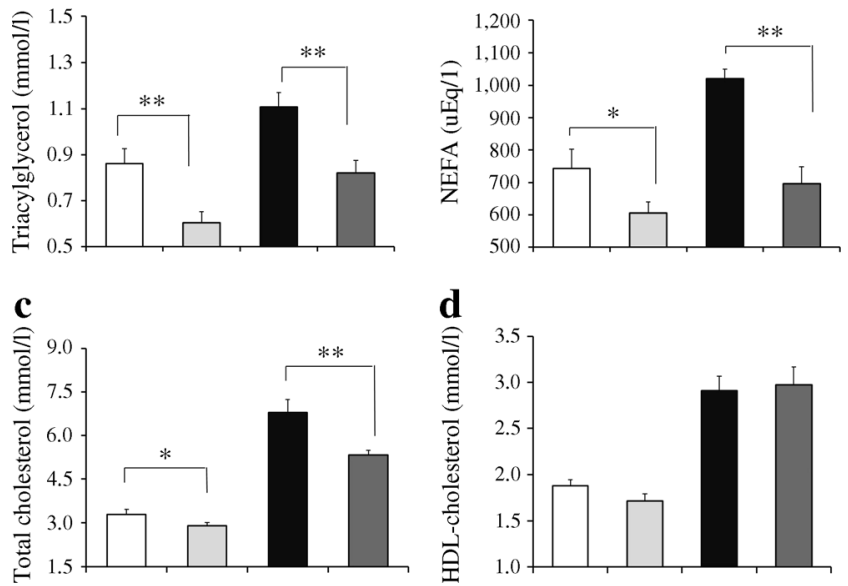

Fig. 3 Il-7r-deficient mice show decreased plasma lipid levels. Plasma concentrations of triacylglycerol (a), NEFA (b), total cholesterol (c) and HDL-cholesterol (d) were measured in male WT and $I l-7 r \mathrm{KO}$ mice. Data are means \pm SEM, $n=8 .{ }^{*} p<0.05$ and ${ }^{* *} p<0.01, I l-7 r$ KO vs WT. White bars, WT chow; black bars, WT HFD; light grey bars, $I l-7 r$ KO chow; dark grey bars, $I l-7 r$ KO HFD

also observed in female $\mathrm{Il}-7 \mathrm{r} \mathrm{KO}$ mice vs their WT counterparts on both chow and HFD (ESM Fig. 2).

II-7r KO mice displayed modulated expression of genes related to adiposity and thermogenesis The mRNA expression of Ras (also known as Reml) and Raf (also known as $Z h x 2$ ) was downregulated in the epiWAT of $I l-7 r \mathrm{KO}$ mice relative to WT controls after HFD feeding (Fig. 4a). Western blotting analysis revealed that the protein level of phosphorylated ERK (p-ERK) was significantly decreased in the epiWAT of $I l-7 r$ KO mice vs WT controls when fed an HFD (Fig. 4b). The ratio of p-ERK to total ERK protein was markedly reduced in $I l-7 r \mathrm{KO}$ mice when compared with their WT littermates on the HFD (Fig. 4b). The expression of genes encoding transcription factors, such as peroxisome proliferator-activated receptor $\gamma 2$ (Ppar $\gamma 2$, also known as Pparg) and CCAAT/enhancer binding protein $\alpha$ (C/ebp $\alpha$ also known as Cebpa), and their targets, fatty acid synthase (Fas), leptin and fatty acid translocase $(\mathrm{Cd} 36)$, were significantly decreased in the epiWAT of $I l-7 r \mathrm{KO}$ mice relative to WT controls on both chow and HFD (Fig. 4c). Furthermore, Il-7r deletion significantly increased the expression of thermogenic genes, such as those encoding peroxisome proliferatoractivated receptor $\gamma$ coactivator $1-\alpha(P g c-1 \alpha$ also known as Ppargcla), cell death-inducing DFFA-like effector a (Cidea), uncoupling protein $1(U c p 1)$, transmembrane protein 26 (Tmem 26) and Cd137 (also known as Tnfrsf9), in both epiWAT (Fig. 4d) and subWAT (Fig. 4e).

Il-7r KO mice were protected from WAT inflammation induced by HFD On the chow diet, there was no difference in plasma levels of the proinflammatory cytokines TNF- $\alpha$, IL-
6 and MCP-1 when comparing $I l-7 r$ KO mice with WT controls (Fig. $5 \mathrm{a}-\mathrm{c}$ ). Under HFD feeding conditions, plasma levels of these proinflammatory cytokines were lower in Il-7r KO mice than in WT controls (Fig. 5a-c). The expression of genes related to proinflammatory cytokines ( $\operatorname{Tn} f \alpha$ [also known as Tnf], Il-6 [Il6], Nf-kb [Nfkbl]) was also significantly decreased in the epiWAT of $I l-7 r \mathrm{KO}$ mice relative to their WT counterparts fed the HFD (Fig. 5d).

The expression of genes relating to M2 markers, mannose receptor 1 ( $M r c 1)$, macrophage galactose $N$-acetyl-galactosamine-specific lectins 1 ( $\mathrm{Mgll}$, also known as Clec10a) and arginase $1(\operatorname{Arg} 1)$, was significantly increased in the epiWAT of Il-7r KO mice vs WT controls on both chow and HFD, whereas the expression of genes related to M1 markers, cluster of differentiation $68(\mathrm{Cd} 68)$ and complement component receptor 4 subunit $(C d 11 c$, also known as Itgax), decreased (Fig. 5e, f). We also observed a substantial decrease in F4/80 immunostaining and fewer crown-like structures in the epiWAT of $I l-7 r$ KO vs WT mice (Fig. $5 \mathrm{~g}$ ).

Il-7r KO mice were protected from HFD-induced insulin resistance An OGTT was performed 2 weeks before the end of the experimental period. Integrated plasma glucose concentration, as calculated by AUC, was decreased in $I l-7 r \mathrm{KO}$ mice compared with their WT counterparts on both chow and HFD (Fig. 6a, b). Fasting plasma glucose and insulin levels were measured at the end of the feeding period. $I l-7 r \mathrm{KO}$ mice fed the HFD exhibited significant reductions in fasting plasma glucose and insulin levels compared with their WT counterparts (Fig. 6c, d). Along with the plasma glucose and insulin levels, the HOMA-IR values indicated that insulin sensitivity was significantly improved in $I l-7 r \mathrm{KO}$ mice maintained on HFD (Fig. 6e).

We found that tyrosine phosphorylation of both IRS1 and Akt increased in epiWAT of $I l-7 r \mathrm{KO}$ mice relative to WT controls on both chow and HFD (Fig. 6f, g). The level of GLUT4 protein found in the membrane fraction of the epididymal adipose tissue was markedly increased in HFD-fed $\mathrm{Il}-7 r$ $\mathrm{KO}$ mice relative to WT mice, whereas the amount of total cellular GLUT4 remained the same when comparing genotypes (Fig. 6h). The ratio of GLUT4 in the plasma membrane to the total amount of GLUT4 was significantly increased in Il-7r KO mice when compared with WT controls on the HFD (Fig. 6h).

\section{Discussion}

The expression of IL-7R was thought to be restricted to haematopoietic cells, primarily B and $\mathrm{T}$ lymphocytes. Levels of IL-7R are tightly regulated during lymphocyte development, with alterations occurring depending on cell maturity and activation status [25]. Cosenza et al [26] demonstrated 
Fig. 4 Il-7r-deficient mice display altered expression of genes related to adipogenesis and thermogenesis. (a) Relative mRNA expression of Ras and Raf in the epiWAT from WT or $I l-7 r$ KO mice. (b) Phosphorylation and expression of ERK in the epiWAT was examined by immunoblot analysis. (c) Relative mRNA expression of adipogenic genes in the epiWAT from WT or Il-7r KO mice. (d, e) Relative mRNA expression of

thermogenic genes in the epiWAT (d) and subWAT (e) from WT or Il-7r KO mice. Data are means \pm SEM, $n=8 .{ }^{*} p<0.05$ and $* * p<0.01, * * * p<0.001 \mathrm{Il}-7 r \mathrm{KO}$ vs WT mice. White bars, WT chow; black bars, WT HFD; light grey bars, $I l-7 r \mathrm{KO}$ chow; dark grey bars, $I l-7 r$ KO HFD $\mathbf{a}$
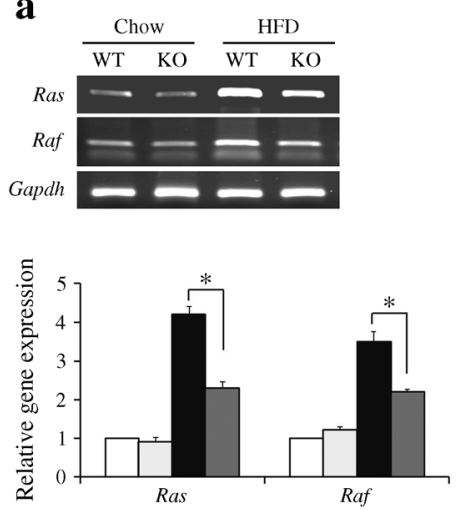
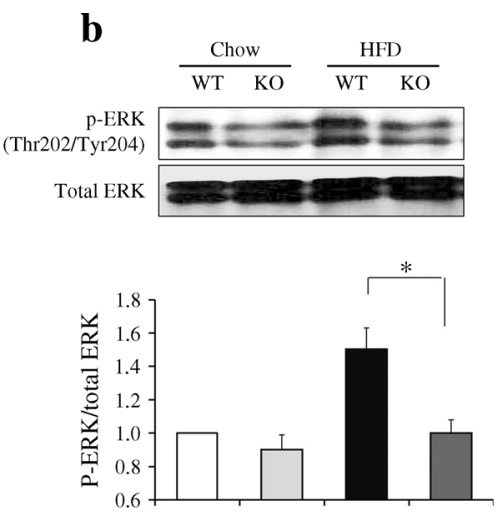

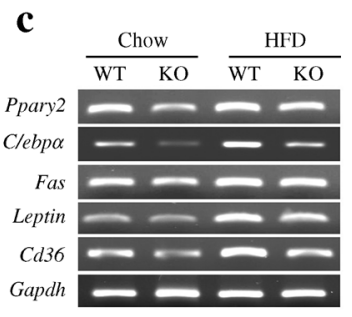

d

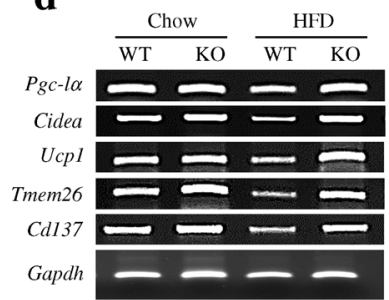

$\mathbf{e}$

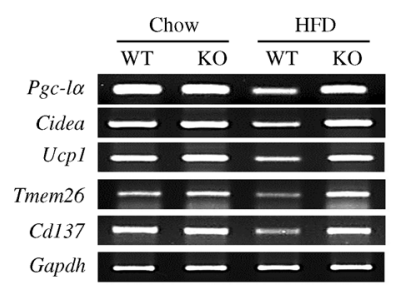

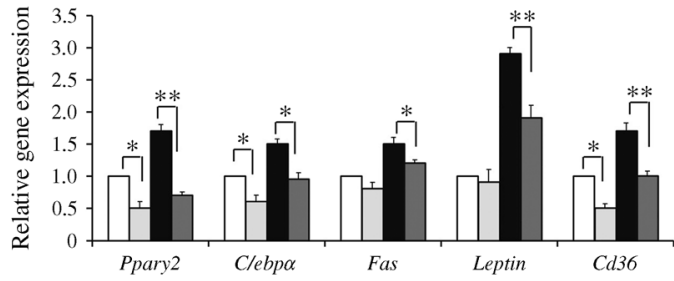
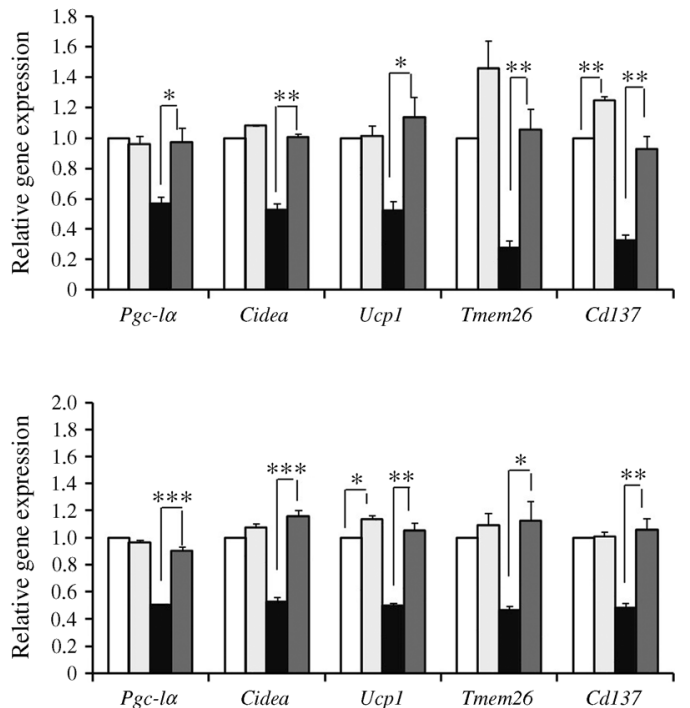

that IL-7R was also expressed in non-haematopoietic cells, including lung, neuronal, breast, renal and colon cell lines. Through cDNA microarray analysis, we previously observed that $I l-7 r$ mRNA was expressed in the WAT of mice, with significantly higher levels in HFD-fed mice than in normal diet-fed mice (T. Park, data not published). These observations prompted us to focus on the role of IL-7R in WAT, related to obesity and metabolic complications.

Oestrogens, the primary female sex hormones, are suggested to reduce visceral adiposity and prevent insulin resistance and glucose intolerance in rodents [27, 28]. For this reason, male mice are more susceptible to diet-induced obesity than female mice. This sex difference was also observed in our present study; female mice exhibited less HFD-induced increase in body weight than male mice (25\% vs 32\%) (ESM Fig. 1). When mice fed an HFD were subjected to glucose tolerance tests, female mice had lower blood glucose levels than male mice at every time point (ESM Fig. 2). Nevertheless, in this study, mice deficient in $I l-7 r$ exhibited a similar extent of decrease in body weight and similar insulin resistance when comparing sexes (ESM Figs 1 and 2). Therefore, the therapeutic effect of IL-7R inhibition in obesity and metabolic diseases could be similar in male and female mice.

It has been reported that stimulation of B cells with IL-7R leads to the activation of the mitogen-activated protein kinase (MAPK)/ERK pathway [29]. ERK is a critical target of IL-7R 
Fig. 5 Il-7r-deficient mice are protected from diet-induced inflammation. (a-c) Plasma concentrations of proinflammatory cytokines. $(\mathbf{d}-\mathbf{f})$ Relative mRNA expression of inflammatory markers in epiWAT. (g) Representative immunohistochemistry images of epiWAT sections stained with F4/80 antibody. Arrows indicate macrophages. Original magnification $\times 40$. Data are means \pm SEM, $n=8 .{ }^{*} p<0.05$ and $* * p<0.01, I l-7 r \mathrm{KO}$ vs WT mice. White bars, WT chow; black bars, WT HFD; light grey bars, $I l-7 r$ KO chow; dark grey bars, $\mathrm{Il}-7 \mathrm{r}$ KO HFD

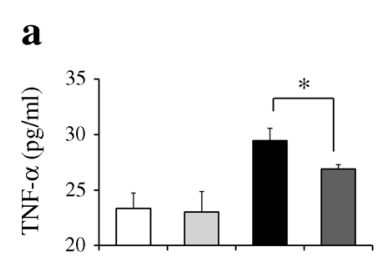

d b

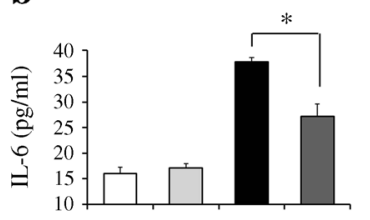

c

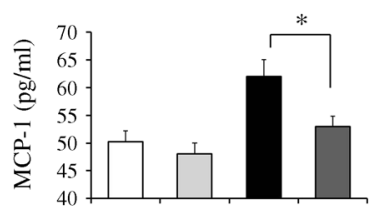

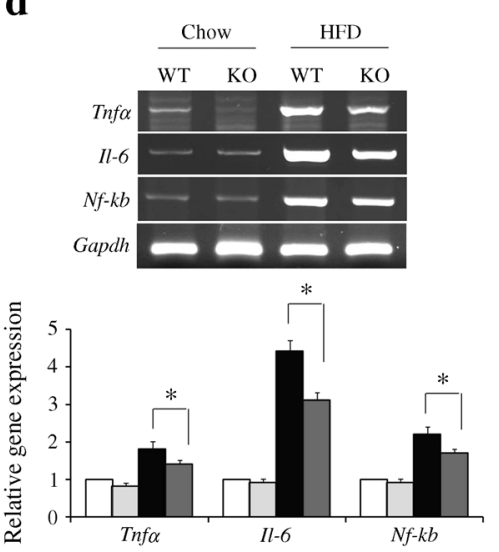

f

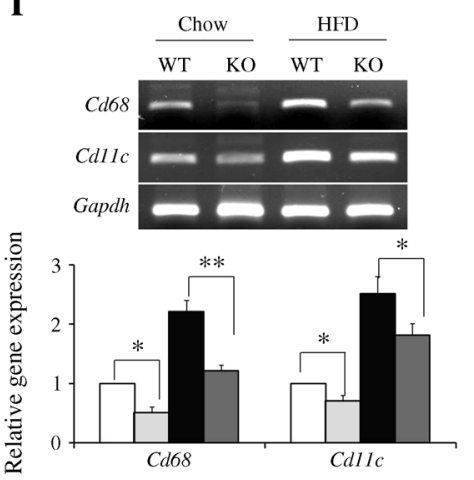

$\mathbf{e}$

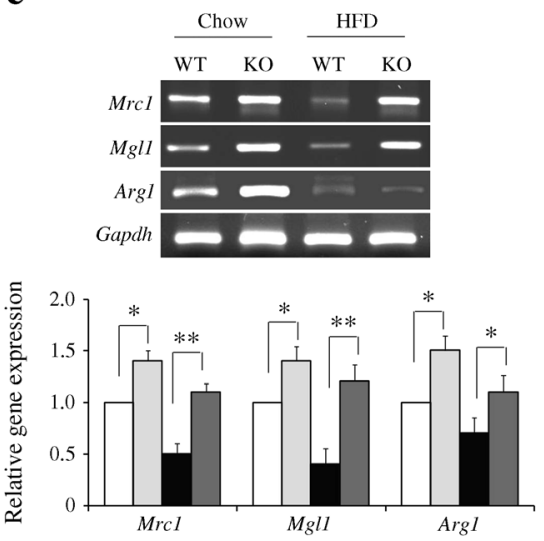

g

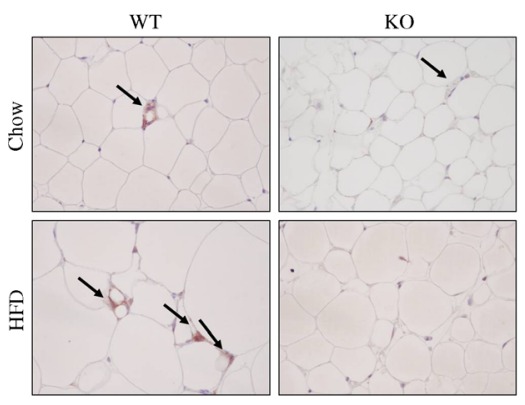

in pre-B cells, as demonstrated by the observation that treatment of cells with ERK inhibitors resulted in the ablation of IL-7-induced cell survival and proliferation [30]. On the other hand, mounting evidence indicates that the activation of ERK promotes adipogenesis by enhancing Ppar 2 and C/ebp $\alpha$ gene expression during the differentiation of 3T3-L1 pre-adipocytes [31]. Compared with WT control cells, mouse embryo fibroblasts and pre-adipocytes isolated from ERK-deficient mice exhibited impaired adipogenesis [32]. In this study, Il-7r expression was significantly increased in the WAT of mice fed the HFD compared with mice fed chow (Fig. 1a). We found that the expression of Ras, Raf and p-ERK was decreased in the epiWAT of $\mathrm{Il}-7 \mathrm{r} \mathrm{KO}$ mice, with lean phenotypes relative to WT controls (Fig. 4a, b). Moreover, Il-7r KO mice displayed reduced gene expression of transcriptional factors (Ppar 2 and Clebp $\alpha$ ) and their targets (Fas, Leptin and Cd36) compared with WT controls (Fig. 4c). Taken together, our findings suggest that IL-7R in WAT may be involved in increased visceral adiposity (Fig. 7). Adipose tissue is made up of several different cell types, including pre-adipocytes, adipocytes, immune cells, endothelial cells, fibroblasts and nerve cells. A further study needs to be conducted in preadipocytes isolated from $\mathrm{Il}-7 \mathrm{r} \mathrm{KO}$ mice in order to prove whether the IL-7R-Ras-Raf-ERK signalling pathway is indeed involved in adipogenesis.

Our findings demonstrate that a lack of $I l-7 r$ protects mice from HFD-induced insulin resistance by improving glucose homeostasis and insulin sensitivity. When a glucose dose is administered based on the total body weight of mice with different fat mass for the evaluation of oral glucose tolerance, the amount of glucose to which the lean tissue, the principal site of glucose disposal, is exposed could be disproportionately low due to decreased fat mass in $I l-7 r \mathrm{KO}$ mice compared with that in obese WT mice with similar lean mass. Therefore, it is plausible that lean mice could be misdiagnosed as being less glucose intolerant simply because they receive less glucose for the same lean body mass. Nevertheless, in this study, fasting blood glucose and insulin levels were also significantly improved in HFD-fed $I l-7 r$ KO mice when compared with their WT counterparts. Mechanistically, IRS-1 
Fig. 6 Deletion of $I l-7 r$ improves diet-induced insulin resistance. (a) Glucose tolerance tests were performed by measuring blood glucose concentrations in mice following an oral load of glucose $(2 \mathrm{~g} / \mathrm{kg})$. (b) AUC was calculated. $(\mathbf{c}, \mathbf{d})$ Blood glucose and insulin concentrations of male WT and Il$7 r$ KO mice. (e) HOMA-IR. (f- $-\mathbf{h})$ Protein levels of p-IRS1, p-Akt, membrane (M) GLUT4 and total (T) GLUT4 in the epiWAT were examined by immunoblot analysis. Quantification of immunoblot data is also shown. Data are means \pm SEM, $n=8$. ${ }^{*} p<0.05, * * p<0.01$ and $* * * p<0.01, \mathrm{Il}-7 r \mathrm{KO}$ vs WT mice. White circles and bars, WT chow; black circles and bars, WT HFD; light grey squares and bars, $I l-7 r$ KO chow; dark grey squares and bars, $I l-7 r$ KO HFD a

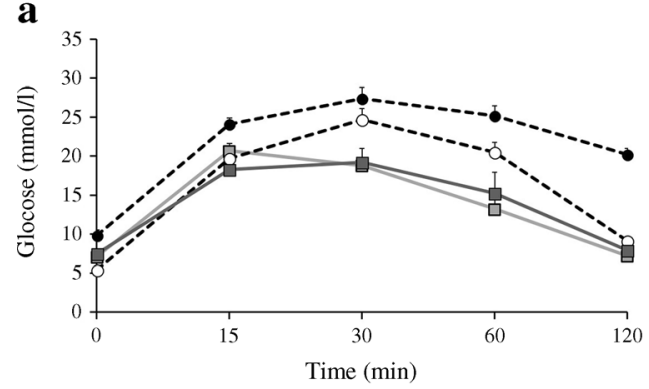

b

c

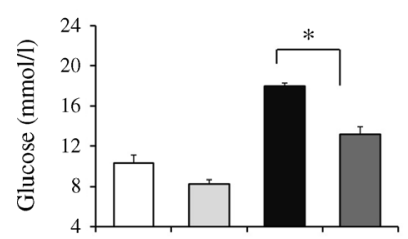

f

d

g
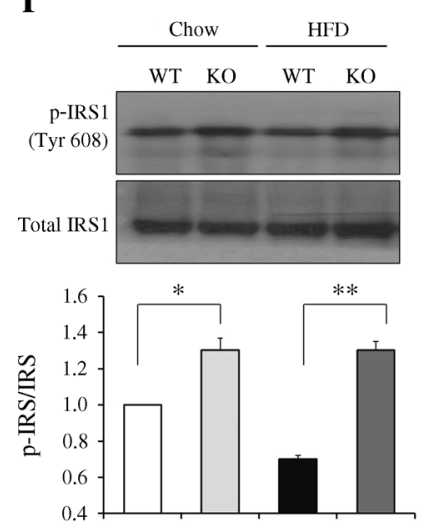
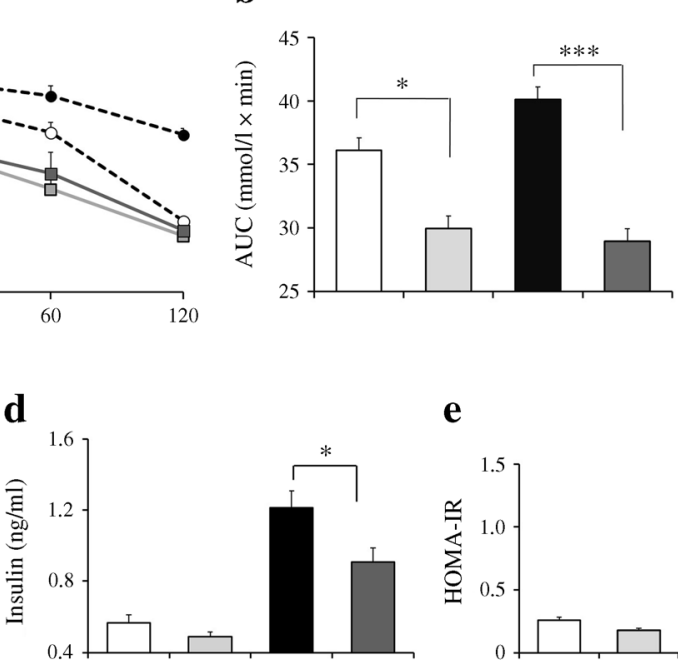

e

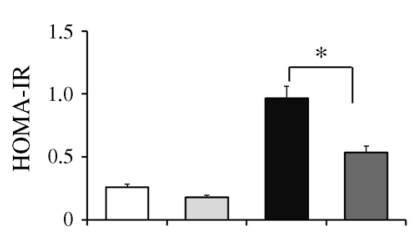

h
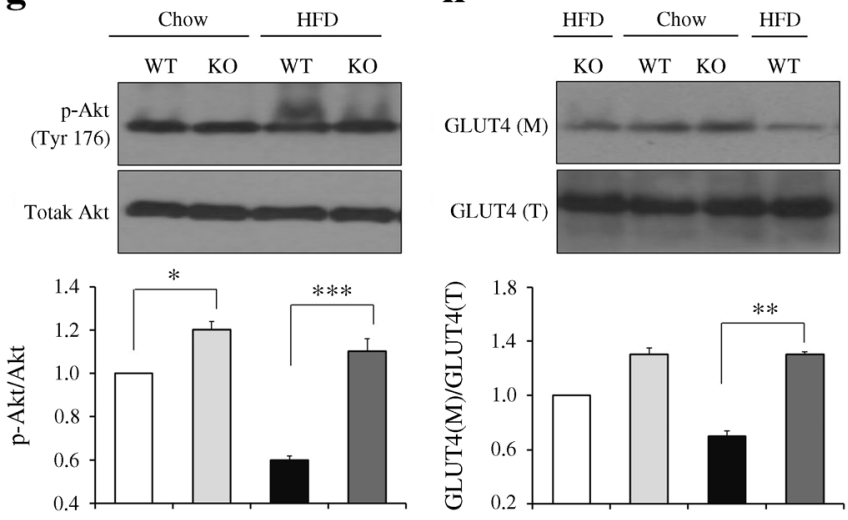

phosphorylation on tyrosine residue stimulates GLUT4 translocation and therefore improves insulin sensitivity [33]. In recent studies, SOCS-1 has been found to cause insulin resistance in obesity by inhibiting tyrosine phosphorylation of IRS-1 in muscle cells and adipocytes [34]. It has been reported that IL-7R signalling involves the recruitment of STAT5, leading to expression of its target gene, Socs 1, in T lymphocyte lineages [35]. In this study, we found that the level of phosphorylated STAT5 and SOCS-1 was reduced in the epiWAT of Il-7r KO mice vs WT controls (Fig. 1b, c). Furthermore, $I l-7 r$ KO mice exhibited increased tyrosine IRS-1 phosphorylation and GLUT4 translocation from the cytoplasm to the membrane compared with WT controls (Fig. 6f, h). Therefore, we suggest that the improvement of insulin sensitivity observed in $\mathrm{Il}-7 r \mathrm{KO}$ mice may be associated with decreased expression of Socs 1, the target gene of the IL-7R-STAT5 pathway in visceral adipocytes (Fig. 7). Moreover, it is well-established that proinflammatory cytokines, such as TNF- $\alpha$ and IL-6, from enlarged visceral adipose tissue impair GLUT4 translocation to the plasma membrane [36]. Despite HFD feeding, Il$7 r \mathrm{KO}$ mice exhibited decreased fat mass and expression levels of proinflammatory cytokines. Thus, improvement of

insulin sensitivity in $I l-7 r \mathrm{KO}$ mice could also be a secondary event followed by decreased fat mass.

Generally, plasma cytokine levels correlate with the expression of proinflammatory adipokines in WAT [37]. In our data, lower levels of plasma proinflammatory cytokines (TNF $\alpha$, IL-6 and MCP1) were observed in $I l-7 r \mathrm{KO}$ mice than in WT controls (Fig. 5a). We also found a marked decrease in the gene expression of proinflammatory cytokines in the WAT of $I l-7 r$ KO mice vs WT controls (Fig. 5d). The ERK-MAPK pathway plays a key regulatory role in the production of proinflammatory cytokines (TNF $\alpha$ and IL-6) by activation of transcriptional factors such as nuclear factor $\mathrm{k}$-lightchain-enhancer of activated B cells in adipocytes [38]. In the present study, $\mathrm{Il}-7 r \mathrm{KO}$ mice exhibited decreased expression of p-ERK and $N f-\kappa b$ in the epiWAT when compared with WT controls (Figs $4 \mathrm{~b}$ and 5d). Consequently, we suggest that the IL-7R-ERK signalling pathway in WAT may be a factor in the initiation and maintenance of obesity-induced peripheral inflammation (Fig. 7). At the same time, it is also probable that the reduction in fat mass caused by $\mathrm{Il}-7 r$ deletion may have contributed to alleviation of WAT inflammation. 


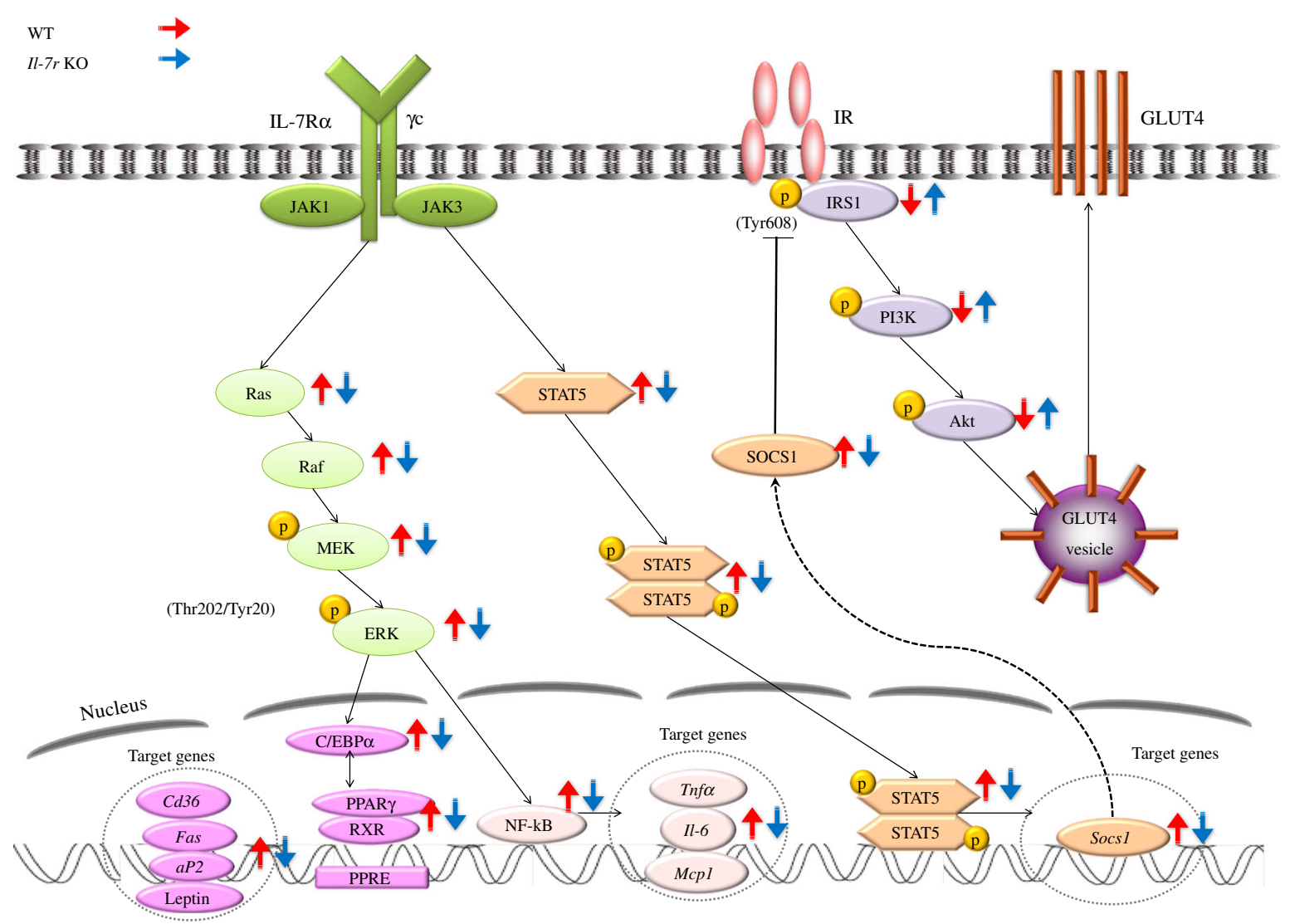

Fig. 7 Schematic presentation of the IL-7R-mediated signalling pathways linked to adipogenesis, inflammation and insulin resistance. $\mathrm{C} / \mathrm{EBP} \alpha$, CCAAT-enhancer-binding protein $\alpha$; MEK, mitogen-activated

Adipose tissue expansion in obesity is associated with infiltration of macrophages that localise to crown-like structures surrounding apoptotic adipocytes [39]. We performed immunohistological staining of adipose tissue for the macrophage marker F4/80 and found markedly fewer macrophages in $I l-7 r \mathrm{KO}$ mice than in WT mice (Fig. $5 \mathrm{~g}$ ). Obesity also leads to a shift in the activation state of macrophages from the M2 anti-inflammatory state to the M1 proinflammatory state [40]. Activated M1 macrophages produce large amounts of proinflammatory mediators, such as TNF $\alpha$ and IL-6, which act on adipocytes to induce an insulin-resistant state [12]. In the present study, expression of M1 marker genes (Cd68 and Cd11c) was dramatically decreased in the WAT of $\mathrm{Il}-7 r \mathrm{KO}$ mice vs WT controls (Fig. 5f), suggesting reduced infiltration of M1 macrophages. Besides the lack of the IL-7R-ERK signalling pathway, the reduction in M1 macrophages is a reasonable mechanism for the decreased WAT inflammation and improved insulin sensitivity observed in $\mathrm{Il}-7 r \mathrm{KO}$ mice.

In conclusion, the present study demonstrates that $\mathrm{Il}-7 \mathrm{r}$ deficiency significantly decreases body and visceral fat pad weights and improves adipose tissue inflammation and insulin sensitivity in mice. These observations implicate IL-7R as a mediator of lipid accumulation in WAT and metabolically triggered inflammation. Further study needs to be done in a protein kinase; PPAR $\gamma$, peroxisome proliferator-activated receptor $\gamma$; $\mathrm{PPRE}$, peroxisome proliferator response element; RXR, retinoid X receptor

conditional knockout mouse model and primary preadipocytes to confirm the role of IL-7R in adipogenesis and insulin resistance in WAT.

Funding This research was supported by the SRC program (Center for Food and Nutritional Genomics: Grant no. 2015R1A5A6001906) of the National Research Foundation of Korea funded by the Ministry of Education, Science and Technology and by a grant of Ministry of Health and Welfare, Republic of Korea (HI13D23470200).

Duality of interest The authors declare that there is no duality of interest associated with this manuscript.

Contribution statement ML and TP designed the study. ML, SJS, MSC, RY and TP participated in acquisition, analysis and interpretation of data. ML and TP drafted the manuscript and ML, SJS, MSC, RY, and TP revised it critically for important intellectual content and approved the final version to be published. TP is the guarantor of this work.

\section{References}

1. Fry TJ, Mackall CL (2005) The many faces of IL-7: from lymphopoiesis to peripheral T cell maintenance. J Immunol 174: 6571-6576

2. Foxwell BM, Beadling C, Guschin D, Kerr I, Cantrell D (1995) Interleukin-7 can induce the activation of Jak 1, Jak 
3 and STAT 5 proteins in murine T cells. Eur J Immunol 25: 3041-3046

3. Pallard C, Stegmann AP, van Kleffens T, Smart F, Venkitaraman A, Spits H (1999) Distinct roles of the phosphatidylinositol 3-kinase and STAT5 pathways in IL-7-mediated development of human thymocyte precursors. Immunity 10:525-535

4. Capitini CM, Chisti AA, Mackall CL (2009) Modulating T cell homeostasis with IL-7: preclinical and clinical studies. J Intern Med 266:141-153

5. Mazzucchelli R, Durum SK (2007) Interleukin-7 receptor expression: intelligent design. Nat Rev Immunol 7:144-154

6. Buckley RH (2004) Molecular defects in human severe combined immunodeficiency and approaches to immune reconstitution. Annu Rev Immunol 22:625-655

7. Puel A, Ziegler SF, Buckley RH, Leonard WJ (1998) Defective IL7R expression in $\mathrm{T}(-) \mathrm{B}(+) \mathrm{NK}(+)$ severe combined immunodeficiency. Nat Genet 20:394-397

8. Hotamisligil GS (2006) Inflammation and metabolic disorders. Nature 444:860-867

9. Scherer PE (2006) Adipose tissue: from lipid storage compartment to endocrine organ. Diabetes 55:1537-1545

10. Fontana L, Eagon JC, Trujillo ME, Scherer PE, Klein S (2007) Visceral fat adipokine secretion is associated with systemic inflammation in obese humans. Diabetes 56:1010-1013

11. Xu H, Barnes GT, Yang Q et al (2003) Chronic inflammation in fat plays a crucial role in the development of obesity-related insulin resistance. J Clin Invest 112:1821-1830

12. Olefsky JM, Glass CK (2010) Macrophages, inflammation, and insulin resistance. Annu Rev Physiol 72:219-246

13. Hotamisligil GS, Shargill NS, Spiegelman BM (1993) Adipose expression of tumor necrosis factor-alpha: direct role in obesitylinked insulin resistance. Science 259:87-91

14. Paz K, Hemi R, LeRoith D et al (1997) A molecular basis for insulin resistance. Elevated serine/threonine phosphorylation of IRS-1 and IRS-2 inhibits their binding to the juxtamembrane region of the insulin receptor and impairs their ability to undergo insulininduced tyrosine phosphorylation. J Biol Chem 272:29911-29918

15. Aguirre V, Uchida T, Yenush L, Davis R, White MF (2000) The cJun $\mathrm{NH}(2)$-terminal kinase promotes insulin resistance during association with insulin receptor substrate-1 and phosphorylation of Ser(307). J Biol Chem 275:9047-9054

16. Serrano-Marco L, Rodriguez-Calvo R, El Kochairi I et al (2011) Activation of peroxisome proliferator-activated receptor- $\beta /$ $\delta($ PPAR $-\beta /-\delta)$ ameliorates insulin signaling and reduces SOCS3 levels by inhibiting STAT3 in interleukin-6 stimulated adipocytes. Diabetes 60:1990-1999

17. Vozarova B, Weyer C, Hanson K, Tataranni PA, Bogardus C, Pratley RE (2001) Circulating interleukin-6 in relation to adiposity, insulin action, and insulin secretion. Obes Res 9:414-417

18. Alderson MR, Tough TW, Ziegler SF, Grabstein KH (1991) Interleukin 7 induces cytokine secretion and tumoricidal activity by human peripheral blood monocytes. J Exp Med 173:923-930

19. Li R, Paul A, Ko KW et al (2012) Interleukin-7 induces recruitment of monocytes/macrophages to endothelium. Eur Heart J 33:3114-3123

20. Hartgring SA, Willis CR, Alcorn D et al (2010) Blockade of the interleukin-7 receptor inhibits collagen-induced arthritis and is associated with reduction of $\mathrm{T}$ cell activity and proinflammatory mediators. Arthritis Rheum 62:2716-2725

21. Hartgring SA, Willis CR, Bijlsma JW, Lafeber FP, van Roon JA (2012) Interleukin-7-aggravated joint inflammation and tissue destruction in collagen-induced arthritis is associated with $\mathrm{T}$ cell and B cell activation. Arthritis Res Ther 14:R137

22. Willis CR, Seamons A, Maxwell J et al (2012) Interleukin-7 receptor blockade suppresses adaptive and innate inflammatory responses in experimental colitis. J Inflamm (Lond) 9:39
23. Ohana M, Okazaki K, Oshima C et al (2001) A critical role for IL7R signaling in the development of Helicobacter felis-induced gastritis in mice. Gastroenterology 121:329-336

24. Maury E, Ehala-Aleksejev K, Guiot Y, Detry R, Vandenhooft A, Brichard SM (2007) Adipokines oversecreted by omental adipose tissue in human obesity. Am J Physiol Endocrinol Metab 293: E656-E665

25. Schluns KS, Lefrancois L (2003) Cytokine control of memory T cell development and survival. Nat Rev Immunol 3:269-279

26. Cosenza L, Gorgun G, Urbano A, Foss F (2002) Interleukin-7 receptor expression and activation in nonhaematopoietic neoplastic cell lines. Cell Signal 14:317-325

27. Zoth N, Weigt C, Laudenbach-Leschowski U, Diel P (2010) Physical activity and estrogen treatment reduce visceral body fat and serum levels of leptin in an additive manner in a diet induced animal model of obesity. J Steroid Biochem Mol Biol 122:100-105

28. Riant E, Waget A, Cogo H, Arnal JF, Burcelin R, Gourdy P (2009) Estrogens protect against high-fat diet-induced insulin resistance and glucose intolerance in mice. Endocrinology 150:2109-2117

29. Fleming HE, Paige CJ (2001) Pre-B cell receptor signaling mediates selective response to IL-7 at the pro-B to pre-B cell transition via an ERK/MAP kinase-dependent pathway. Immunity 15: $521-531$

30. Yasuda T, Sanjo H, Pages G et al (2008) Erk kinases link pre-B cell receptor signaling to transcriptional events required for early B cell expansion. Immunity 28:499-508

31. Prusty D, Park BH, Davis KE, Farmer SR (2002) Activation of MEK/ERK signaling promotes adipogenesis by enhancing peroxisome proliferator-activated receptor gamma (PPAR $\gamma)$ and $\mathrm{C} / \mathrm{EBP} \alpha$ gene expression during the differentiation of $3 \mathrm{~T} 3-\mathrm{L} 1$ preadipocytes. J Biol Chem 277:46226-46232

32. Bost F, Aouadi M, Caron L et al (2005) The extracellular signalregulated kinase isoform ERK1 is specifically required for in vitro and in vivo adipogenesis. Diabetes 54:402-411

33. Morris AJ, Martin SS, Haruta T et al (1996) Evidence for an insulin receptor substrate 1 independent insulin signaling pathway that mediates insulin-responsive glucose transporter (GLUT4) translocation. Proc Natl Acad Sci U S A 93:8401-8406

34. Ueki K, Kondo T, Kahn CR (2004) Suppressor of cytokine signaling 1 (SOCS-1) and SOCS-3 cause insulin resistance through inhibition of tyrosine phosphorylation of insulin receptor substrate proteins by discrete mechanisms. Mol Cell Biol 24:5434-5446

35. Cornish AL, Chong MM, Davey GM et al (2003) Suppressor of cytokine signaling-1 regulates signaling in response to interleukin-2 and other gamma c-dependent cytokines in peripheral T cells. J Biol Chem 278:22755-22761

36. Leguisamo NM, Lehnen AM, Machado UF et al (2012) GLUT4 content decreases along with insulin resistance and high levels of inflammatory markers in rats with metabolic syndrome. Cardiovasc Diabetol 11:100

37. Gregor MF, Hotamisligil GS (2011) Inflammatory mechanisms in obesity. Annu Rev Immunol 29:415-445

38. Chung S, Lapoint K, Martinez K, Kennedy A, Boysen Sandberg M, McIntosh MK (2006) Preadipocytes mediate lipopolysaccharideinduced inflammation and insulin resistance in primary cultures of newly differentiated human adipocytes. Endocrinology 147:5340-5351

39. Cinti S, Mitchell G, Barbatelli G et al (2005) Adipocyte death defines macrophage localization and function in adipose tissue of obese mice and humans. J Lipid Res 46:2347-2355

40. Lumeng CN, Bodzin JL, Saltiel AR (2007) Obesity induces a phenotypic switch in adipose tissue macrophage polarization. J Clin Invest 117:175-184 